\title{
A NOTE ON A METHOD OF MILNE-THOMSON
}

\author{
V. T. BUCHWALD
}

(received 24 July 1962)

\begin{abstract}
Milne-Thomson has used the method of analytic continuation to solve boundary value problems of the annulus in plane elastostatics. However, his use of Cauchy integrals is incorrect, and it is shown in this note that the solution is obtained in terms of Laurent Series expansions. The solution is equivalent to that of Muskhelishvili, but is simpler to use in some applications.

A similar approach is used to solve the boundary value problem of the infinite strip, the solution being given in terms of functions of a complex variable expressed as Fourier integrals.
\end{abstract}

\section{The Annulus}

The double Laurent expansion solution of the plane elastostatic boundary value problems of the annulus is given by Muskehlishvili [1]. More recently, Milne-Thomson [2] has used an analytic continuation method to reformulate the boundary value problem. However, while his method is interesting and important, his way of obtaining a closed solution afterwards is incorrect. It is shown here that the analytic continuation approach gives a series solution, similar to, but somewhat simpler than that obtained by the Muskhelishvili method. We shall confine ourselves to the first boundary value problem of plane strain, as results for other boundary value problems are entirely analogous.

Consider the annulus $S$ in the region $a<|z|<b$, where $z=x+i y$, and assume that

$$
\begin{aligned}
\tau_{r r}+i \tau_{r \theta} & =f(\theta), \quad z=\sigma=b e^{i \theta}, \\
& =g(\theta), \quad z=\tau=a e^{i \theta},
\end{aligned}
$$

where the normal and tangential stresses $\tau_{r r}, \tau_{r \theta}$ can be expressed in terms of two functions of a complex variable, analytic in $S$, by

$$
\tau_{r \tau}+i \tau_{r \theta}=W(z)+\bar{W}(\bar{z})-\bar{z} \bar{W}^{\prime}(\bar{z})-\frac{\bar{z}}{z} \bar{w}(\bar{z}) .
$$


Let $S^{-}, S^{+}$be the annuli $a^{2} / b<|z|<a, b<|z|<b^{2} / a$, respectively; $S^{-}$is the inverse of $S$ with respect to the circle $z=\tau$, and $S^{+}$its inverse with respect to $z=\sigma$. Assume the analytic continuation of $W(z)$ in $S^{-}$is

$$
W(z)=-\bar{W}\left(a^{2} / z\right)+\frac{a^{2}}{z} \bar{W}^{\prime}\left(a^{2} / z\right)+\frac{a^{2}}{z^{2}} \bar{w}\left(a^{2} / z\right), \quad z \text { in } S^{-},
$$

so that $W(z)$ is analytic in $S^{-}$. Hence

$$
w(z)=\frac{a^{2}}{z^{2}}\left\{\bar{W}\left(a^{2} / z\right)+W(z)-z W^{\prime}(z)\right\}, \quad z \text { in } S,
$$

and, from (2)

(5) $\quad \tau_{r r}+i \tau_{r \theta}=W(z)-\frac{a^{2}}{z \bar{z}} W\left(a^{2} / \bar{z}\right)+\left(1-a^{2} / z \bar{z}\right)\left\{\bar{W}(\bar{z})-\bar{z} \bar{W}^{\prime}(\bar{z})\right\}, \quad z$ in $S$.

Thus, when $z=\tau$

$$
W(\tau)-W_{-}(\tau)=g(\theta)
$$

where $W_{-}(\tau)$ is the value of $W(\tau)$ as $z$ approaches $\tau$ in $S^{-}$.

Similarly, defining the analytic continuation of $W(z)$ in $S^{+}$by

$$
W(z)=-\bar{W}\left(b^{2} / z\right)+\frac{b^{2}}{z} \bar{W}^{\prime}\left(b^{2} / z\right)+\frac{b^{2}}{z^{2}} \bar{w}\left(b^{2} / z\right), \quad z \text { in } S^{+}
$$

so that

$$
w(z)=\frac{b^{2}}{z^{2}}\left\{\bar{W}\left(b^{2} / z\right)+W(z)-z W^{\prime}(z)\right\}, \quad z \text { in } S,
$$

whence

$$
W(\sigma)-W_{+}(\sigma)=f(\theta),
$$

where $W_{+}(\sigma)$ is the value of $W(z)$ as $z$ approaches $\sigma$ in $S^{+}$.

The function $w(z)$ has been defined in two different ways in equation (4) and $(8)$, so that, for compatibility,

$$
b^{2} \bar{W}\left(b^{2} / z\right)-a^{2} \bar{W}\left(a^{2} / z\right)+\left(b^{2}-a^{2}\right)\left\{W(z)-z W^{\prime}(z)\right\}=0 .
$$

Equations (6), (9) and (10) are those obtained by Milne-Thomson [2], and are sufficient to determine $W(z)$ and hence $w(z)$. However, at this stage Milne-Thomson expressed $W(z)$ in terms of Cauchy integrals, using equations (6) and (9). In the general case this is not valid, since the Plemej formulae, as used by him, would only apply if the region $S^{+}$were extended to infinity, and the region $S^{-}$to the origin. There is no reason to believe that $W(z)$ can be continued in a simple way outside the region $a^{2} / b \leqq|z| \leqq b^{2} / a$, and the Cauchy integral approach breaks down. 
The correct approach is to expand $W(z)$ in a Laurent series in each of the three regions by

$$
\begin{aligned}
W(z) & =\sum_{-\infty}^{\infty} A_{n}^{+} z^{n}, \quad z \text { in } S^{+}, \\
& =\sum_{-\infty}^{\infty} A_{n} z^{n}, \quad z \text { in } S, \\
& =\sum_{-\infty}^{\infty} A_{n}^{-} z^{n}, \quad z \text { in } S^{-} .
\end{aligned}
$$

From (6) and (9), respectively, we find that

$$
A_{n}-A_{n}^{-}=g_{n} / a^{n},
$$

and

$$
A_{n}-A_{n}^{+}=f_{n} / b^{n},
$$

where $g_{n}, f_{n}$, are the Fourier coefficients given by

$$
f_{n}=\frac{1}{2 \pi} \int_{0}^{2 \pi} f(\theta) e^{-i n \theta} d \theta ; \quad g_{n}=\frac{1}{2 \pi} \int_{0}^{2 \pi} g(\theta) e^{-i n \theta} d \theta .
$$

Also, using (14) and equating to zero the coefficient of $z^{n}$ in equation (10)

$$
\left(b^{2}-a^{2}\right)(1-n) A_{n}+b^{2(1-n)} A_{-n}^{+}-a^{2(1-n)} A_{n}^{-}=0 .
$$

Using the relations (12) and (13) for $A_{n}^{+}, A_{n}^{-}$in terms of $A_{n}$, we deduce that

$$
\left(b^{2}-a^{2}\right)(1-n) A_{n}+\left\{b^{2(1-n)}-a^{2(1-n)}\right\} A_{-n}=b^{2-n} \bar{f}_{-n}-a^{2-n} \bar{g}_{-n} .
$$

A similar equation can be found by equating to zero the coefficient of $z^{-n}$ in (10), and this can also be obtained by taking the complex conjugate of (16) and replacing $n$ by $-n$, when

$$
\left\{b^{2(1+n)}-a^{2(1+n)}\right\} A_{n}+\left(b^{2}-a^{2}\right)(1+n) \bar{A}_{-n}=b^{2+n} f_{n}-a^{2+n} g_{n} .
$$

Eliminating $\bar{A}_{-n}$ from (16) and (17) we find, formally,

$$
\begin{aligned}
\Delta W(z)= & \left(b^{2}-a^{2}\right)(1+n)\left(b^{2-n} \bar{f}_{-n}-a^{2-n} \bar{g}_{-n}\right) \\
& -\left(b^{2-2 n}-a^{2-2 n}\right)\left(b^{2+n} f_{n}-a^{2+n} g_{n}\right),
\end{aligned}
$$

where

$$
\Delta=\left(b^{2}-a^{2}\right)^{2}\left(1-n^{2}\right)-\left\{b^{2(1-n)}-a^{2(1-n)}\right\}\left\{b^{2(1+n)}-a^{2(1+n)}\right\} .
$$

The function $w(z)$ can now be determined by substituting the expression (18) for $W(z)$ in either of the equations (4) or (8). Equation (18) holds for all $n$ other than $n=0, \pm 1$, when $\Delta \equiv 0$. Here we have to use the conditions of static equilibrium on the annulus, obtained by assuming (i) zero resultant force, and (ii) zero moment, which respectively imply 

(i) $b f_{-1}-a g_{-1}=0$; and
(ii) $\operatorname{Im}\left\{b^{2} f_{0}-a^{2} g_{0}\right\}=0$.

Equation (16) is now satisfied identically in the case $n=1$, and, for $n=0$,

$$
2\left(b^{2}-a^{2}\right) \operatorname{Re}\left(A_{0}\right)=b^{2} f_{0}-a^{2} g_{0} .
$$

If $W(z)=i C$, where $C$ is a real constant, then $w(z)=0$, all the stresses are zero, and the displacements are merely those of a rigid body. Hence $\operatorname{Im}\left(A_{0}\right)$ is arbitrary. Also, the condition for single valued displacements is that $\int_{0}^{2 \pi} W(z) d z=0$, so that $A_{-1}$ vanishes. The coefficient $A_{1}$ can now be obtained by making $n=1$ in (17), yielding

$$
\left(b^{4}-a^{4}\right) A_{1}=b^{3} f_{1}-a^{3} g_{1} .
$$

Equations (18), (20), (21) together with (4) give the solution to the problem, and are equivalent to Muskhelishvili's results, the only advantage being that it is not necessary to expand $w(z)$ in a Laurent series, since it is given in terms of $W(z)$ by either (4) or (8). In practical cases the number of unknowns is halved, and we only need to compute the $A_{n}$.

If we assume $g(\theta)=0$, we can adopt an even simpler procedure, since, from (12), $A_{n}=A_{n}^{-}$. We now only need to consider equations (3), (4), (5), in which equation (3) gives the unique continuation of $W(z)$ from $S$ into $S^{-}$, $W(z)$ being continuous across the circle $z=\tau$. Putting $z=\sigma$ in (5), and using $\bar{\sigma}=b^{2} / \sigma$, we find that

$$
W(\sigma)-\frac{a^{2}}{b^{2}} W\left(a^{2} / b^{2} \sigma\right)+\left(1-\frac{a^{2}}{b^{2}}\right)\left\{\bar{W}\left(b^{2} / \sigma\right)-\frac{b^{2}}{\sigma} \bar{W}^{\prime}\left(b^{2} / \sigma\right)\right\}=f(\sigma / b) .
$$

Expanding $W(z)$ in a Laurent series, the coefficients can be evaluated by comparing coefficients of $\sigma^{n}$ in equation (22), with results similar to the ones already derived, but with $g_{n}=0$. This latter approach has been used by Davies [3] for certain doubly connected regions with reinforced boundaries, and also by Buchwald and Davies [4], for a simple numerical solution of the case of a circular hole in an elliptic plate, and also for an anisotropic annular plate.

\section{The infinite strip}

Boundary value problems of the infinite strip have been solved by Filon [5] Hopkins [6], inter alia, using the Airy stress function and Fourier integrals. At best, this approach is algebraically involved, and Tiffen $[7,8]$ has used a complex variable approach, but his method is unduly complicated. The Milne-Thomson technique gives a very simple theory.

Let the strip occupy the region $S,-1<y<1$, and the regions $S^{+}, S^{-}$ are $1<y<3$, and $-3<y<-1$, respectively. The stresses $\tau_{y y}, \tau_{x y}$ 
are given in terms of complex potentials by, (cf. Milne-Thomson, loc.cit. p. 87),

$$
\tau_{y y}-i \tau_{x y}=W(z)+\bar{W}(\bar{z})+z \bar{W}^{\prime}(\bar{z})+\bar{w}(\bar{z}) .
$$

Assume that we are given that

$$
\begin{aligned}
\tau_{y y}-i \tau_{x y} & =f(x), & & y=1, \\
& =g(x), & & y=-1,
\end{aligned}
$$

and we need to find $W(z), w(z)$ which satisfy $(24)$ on the boundaries.

Define the analytic continuation of $W(z)$ in $S^{+}$by

$$
W(z)=-z \bar{W}^{\prime}(z-2 i)-\bar{W}(z-2 i)-\bar{w}(z-2 i), \quad z \text { in } S^{+},
$$

so that

$$
w(z)=-W(z)-(z-2 i) W^{\prime}(z)-\bar{W}(z-2 i), \quad z \text { in } S,
$$

whence, from (23) and (26),

$$
W(x+i)-W_{+}(x+i)=f(x),
$$

where $W^{+}(x+i)$ is the limit of $W(z)$ as $z$ tends to $x+i$, in $S^{+}$. Similarly, define

$$
W(z)=z \bar{W}^{\prime}(z+2 i)-\bar{W}(z+2 i)-\bar{w}(z+2 i), \quad z \text { in } S^{-}
$$

whence

$$
w(z)=-W(z)-(z+2 i) W^{\prime}(z)-\bar{W}(z+2 i), \quad z \text { in } S,
$$

and, from (23) and (26)

$$
W(x-i)-W_{-}(x-i)=g(x) .
$$

The condition that the definitions (26) and (29) are compatible is that

$$
\bar{W}(z+2 i)-\bar{W}(z-2 i)+4 i W^{\prime}(z)=0 .
$$

Equations (27), (30) and (31) are sufficient to determine $W(z)$ and can be solved by the Fourier Integral method used by Titchmarsh [9] for differential-difference equations. Assume that $W(z)$ can be expressed as the Fourier integral

$$
W(z)=\int_{-\infty}^{\infty} \phi(t) e^{-i z t} d t, \quad z \text { in } S,
$$

with $\phi_{+}(t), \phi_{-}(t)$ being the Fourier transforms of $W(z)$ with $z$ in $S^{+}$and $S^{-}$, respectively. Taking the transform of equations (27) and (30), 
$(33)$

$$
\phi(t)-\phi_{+}(t)=F(t) e^{-t},
$$

and

$$
\phi(t)-\phi_{-}(t)=G(t) e^{t},
$$

where $2 \pi F(t)=\int_{-\infty}^{\infty} f(x) e^{i x t} d x$; and $2 \pi G(t)=\int_{-\infty}^{\infty} g(x) e^{i x t} d x$. Also, taking the transform of (31), we find, after some reduction,

$$
e^{2 t} \bar{\phi}_{-}(-t)-e^{-2 t} \bar{\phi}_{+}(-t)+4 t \phi(t)=0 .
$$

Combining those equations,

$$
\sinh 2 t \bar{\phi}(-t)+2 t \phi(t)=-\bar{H}(-t),
$$

where $H(t)=\frac{1}{2}\left\{e^{t} F(t)-e^{-t} G(t)\right\}$. Taking the complex conjugate of (35), and replacing $t$ by $-t$,

$$
2 t \bar{\phi}(-t)+\sinh 2 t \phi(t)=H(t) .
$$

Eliminating $\Phi(-t)$, we find that $\phi(t)$ is given by

$$
\left(\sinh ^{2} 2 t-4 t^{2}\right) \phi(t)=\sinh 2 t H(t)+2 t \bar{H}(-t) .
$$

Equations (37), (32) and either of (26) or (29) give the solution of the problem.

\section{References}

[1] N. I. Muskhelishvili, Some Basic problems of the mathematical theory of elasticity, chapter 10 (Translated by S. R. M. Radok, Noordhoff, Groningen, 1953).

[2] L. M. Milne-Thomson, Plane Elastic Systems (Springer-Verlag, Berlin, 1960) p. 130.

[3] G. A. O. Davies, in preparation.

[4] V. T. Buchwald and G. A. O. Davies, Quart. J. Mech. Appl. Math., in the press.

[5] L. N. G. Filon, Phil. Trans. A 201 (1903) 63.

[6] H. G. Hopkins, Proc. Camb. Phil. Soc., 46 (1949) 164.

[7] R. Tiffen, Quart. J. Mech. appl. Math. 6, (1953), 344.

[8] R. Tiffen, Quart. J. Mech. appl. Math. 8, (1955), 237.

[9] Titchmarsh, Theory of Fourier Integrals, $\S 10.16$, (Oxford University Press, 1937).

Department of Applied Mathematics, University of Sydney. 